\title{
External Compton emission from relativistic jets in Galactic black hole candidates and ultraluminous $\mathrm{X}$-ray sources
}

\author{
M. Georganopoulos, F. A. Aharonian, and J. G. Kirk \\ Max-Planck-Institut für Kernphysik, Postfach 1039 80, 69029 Heidelberg, Germany \\ Received 16 October 2001 / Accepted 12 April 2002

\begin{abstract}
Galactic binary systems that contain a black hole candidate show evidence of radio jets in their hard X-ray states. Unavoidably, photons from the companion star and/or the accretion disk are Compton-scattered by relativistic electrons in the jet, producing beamed X-rays and possibly gamma-rays. The importance of this process depends on the jet power and the Doppler boosting factor. For plausible values of these parameters, we show that the jet emission can contribute significantly to the hard state X-ray luminosity. Two sources - XTE J1118+480 and Cygnus X-1 - are modelled as representatives of black holes with low and high luminosity companion stars respectively. In XTE J1118+480, weak reflection features indicate that the jet emission is comparable to coronal emission. In Cygnus X-1, strong reflection features indicate coronal emission in the X-ray band, but the jet emission may emerge in the gamma-ray band. The absence of reflection features in the spectra of the ultraluminous compact $\mathrm{X}$-ray sources in nearby galaxies suggests that they are dominated by jet emission. We show that a viable model for these sources is a stellar mass black hole with a high luminosity companion and a favourably oriented jet.
\end{abstract}

Key words. X-rays: binaries - stars: individual: Cygnus X-1, XTE J1118+480 - radiation mechanisms: non-thermal

\section{Introduction}

Black hole candidates in X-ray binary systems emit in two $\mathrm{X}$-ray states. In the high-soft state the spectral energy distribution $(S E D)$ peaks at a few $\mathrm{keV}$, above which there is a soft power law component. In the low-hard state a hard power law extends to at least $100 \mathrm{keV}$. Both states have been modelled using the comptonisation of accretion disk photons from a hot corona containing a hybrid plasma of thermal and nonthermal electrons (e.g. Zdziarski 2000).

A tight temporal correlation between the low-hard $\mathrm{X}$-ray state and the radio emission has been found (Corbel et al. 2000) and radio jets have been detected in several of these sources, collectively called microquasars (Mirabel \& Rodriguez 1999). In Cygnus X-1 an one-sided relativistic jet has recently been resolved while the source was in the low-hard X-ray state (Stirling et al. 2001). There is increasingly strong evidence that powerful, possibly relativistic jets, with a minimum jet power $L_{\text {inj }} \sim 10^{-3}-10^{-2} L_{\mathrm{E}}$ of the Eddington luminosity $L_{\mathrm{E}}$ may be a generic feature of the lowhard state of microquasars (Fender 2001a). The actual jet power can be much higher than this lower limit (Fender 2001a), as is the case with GRS 1915+105, where $L_{\text {inj }} \gtrsim 0.3 L_{\mathrm{E}}$ (Fender \& Pooley 2000 ). The radio emission of microquasars is synchrotron radiation from the

Send offprint requests to: M. Georganopoulos, e-mail: markos@mickey.mpi-hd.mpg.de jets (Hjellming \& Johnston 1988). Recently it has been proposed that the synchrotron emission might extend up to X-ray energies, either in the extended jet structure (Atoyan \& Aharonian 1999) or close to the base of the jet (Markoff et al. 2001). Also, external Compton scattering $(E C S)$ by relativistic electrons in the jets has been previously proposed as a mechanism for the production of X-rays and gamma-rays (Band \& Grindlay 1986; Levinson \& Blandford 1996; Atoyan \& Aharonian 1999).

Here, using basic observables, we show that, in the low-hard state, ECS of photons from the companion star and/or the accretion disk by relativistic electrons can contribute significantly, or dominate the X-ray flux. We apply this idea to two sources typical of systems with low and high mass companion stars, and show that the compact ultraluminous X-ray sources $(U L X)$ observed in nearby galaxies (Makishima et al. 2000) display the properties of beamed microquasars with high mass companion stars.

\section{ECS in the low-hard state}

Consider relativistic plasma injected at a jet inlet of radius $R$. The plasma flows with a bulk Lorentz factor $\Gamma$ and velocity $\beta c$, where $c$ is the speed of light. For simplicity, assume that, in the comoving frame, electrons are injected isotropically with a power-law distribution of index $p$ confined between Lorentz factors $\gamma_{1}$ and $\gamma_{2}$. The environment is permeated by an isotropic monoenergetic 
photon field of photon energy $\epsilon_{0}$ in units of $m_{\mathrm{e}} c^{2}$ and energy density $U$. The plasma suffers inverse Compton losses until it has reached a distance $l$ from the inlet at which the radiation field drops off significantly. The average time spent by electrons in this part of the jet - the escape time $t_{\text {esc }}$ - is parameterised in units of the light crossing time $t_{\text {esc }}=k l / c$, where $k \gtrsim 1$. The photon energy density $U$ is taken to be constant within the emission region. The comoving photon density in the flow frame can be written as $U^{\prime} \approx \Gamma^{2} U \approx \Gamma^{2} L_{\mathrm{s}} / 4 \pi c d^{2}$, where $L_{\mathrm{s}}$ is the luminosity of the dominant photon source and $d$ is the distance of the emitting plasma from this source. Assuming that Compton losses dominate, the Lorentz factor $\gamma_{\mathrm{b}}$ at which the cooling and escape times are equal is

$\gamma_{\mathrm{b}} \approx \frac{3 m_{\mathrm{e}} c}{4 \sigma_{\mathrm{T}} U^{\prime} t_{\mathrm{esc}}}=\frac{3 \pi m_{\mathrm{e}} c^{3} d^{2}}{\sigma_{\mathrm{T}} k l L_{\mathrm{s}} \Gamma^{2}}$.

The electron kinetic power $L_{\mathrm{inj}}$ injected in the jet is

$L_{\text {inj }}=\frac{4 \pi}{3} R^{2} Q \Gamma^{2} \beta m_{\mathrm{e}} c^{3} \frac{\gamma_{2}^{2-p}-\gamma_{1}^{2-p}}{2-p}$,

where $Q$ is the normalisation constant of the electron energy distribution. For a continuous source, the spectrum of ECS for energies $\epsilon_{\mathrm{b}} \leq \epsilon \leq \epsilon_{\max }$, where $\epsilon_{\mathrm{b}}=4 \epsilon_{0} \gamma_{\mathrm{b}}^{2} \mathcal{D}^{2}$, $\epsilon_{\max }=4 \epsilon_{0} \gamma_{2}^{2} \mathcal{D}^{2}$, is (Georganopoulos et al. 2001)

$\frac{\mathrm{d} L}{\mathrm{~d} \epsilon \mathrm{d} \Omega} \approx \mathcal{D}^{3+p} \frac{Q k \gamma_{\mathrm{b}} V \sigma_{\mathrm{T}} c U 2^{p}}{\pi \epsilon_{0}(2+p)(4+p)}\left(\frac{\epsilon}{\epsilon_{0}}\right)^{-p / 2}$

where $V=\pi R^{2} l$ is the volume of the source, and $\mathcal{D}=$ $1 / \Gamma(1-\beta \cos \theta)$ is the Doppler factor for an observing angle $\theta$. For $p<2$ the luminosity per logarithmic energy interval at the peak energy $\epsilon_{\text {peak }} \approx \epsilon_{0} \mathcal{D}^{2} \gamma_{2}^{2}$ of the SED given by $\epsilon \mathrm{d} L / \mathrm{d} \epsilon \mathrm{d} \Omega$ scales as $\mathcal{D}^{5}$ (Dermer 1995):

$L_{\text {peak }}=4 \pi\left(\frac{\epsilon \mathrm{d} L}{\mathrm{~d} \epsilon \mathrm{d} \Omega}\right)_{\epsilon=\epsilon_{\text {peak }}} \approx \frac{3 \pi m_{\mathrm{e}} c^{3} 2^{p} Q R^{2} \gamma_{2}^{2-p} \mathcal{D}^{5}}{(2+p)(4+p) \Gamma^{2}}$.

\subsection{A microquasar with a low mass companion}

We focus now on the hard X-ray state of XTE J1118+480, a microquasar consisting of a black hole of mass $M \approx$ $6.5 M_{\odot}$ and a K7V-M0V star of luminosity $L_{\mathrm{s}} \approx 4 \times$ $10^{32} \mathrm{erg} \mathrm{s}^{-1}$ separated by $R_{\mathrm{s}} \approx 1.7 \times 10^{11} \mathrm{~cm}$ (McClintock et al. 2001b). The luminosity of the accretion disk is $L_{\mathrm{d}} \approx 8.6 \times 10^{35} \mathrm{erg} \mathrm{s}^{-1}$, with a peak photon energy $\approx 24 \mathrm{eV}$ (McClintock et al. 2001b). The practically absent reflection features of this system led Miller et al. (2002) to suggest that the X-ray emission arises from a jet, and the source has been modeled by Markoff et al. (2001) as a synchrotron X-ray emitting jet of $L_{\mathrm{inj}} \approx 0.02 L_{\mathrm{E}}$.

The accretion disk is much brighter than the companion star. Assuming that the emission site is located on the axis of the accretion disk, perpendicular to the orbital plane of the binary, the photon energy density at the emission site is dominated by accretion disk photons. The X-ray spectral index is $\alpha \approx 0.8$ and the SED peaks at $\sim 100 \mathrm{keV}$. To match these observations, we set

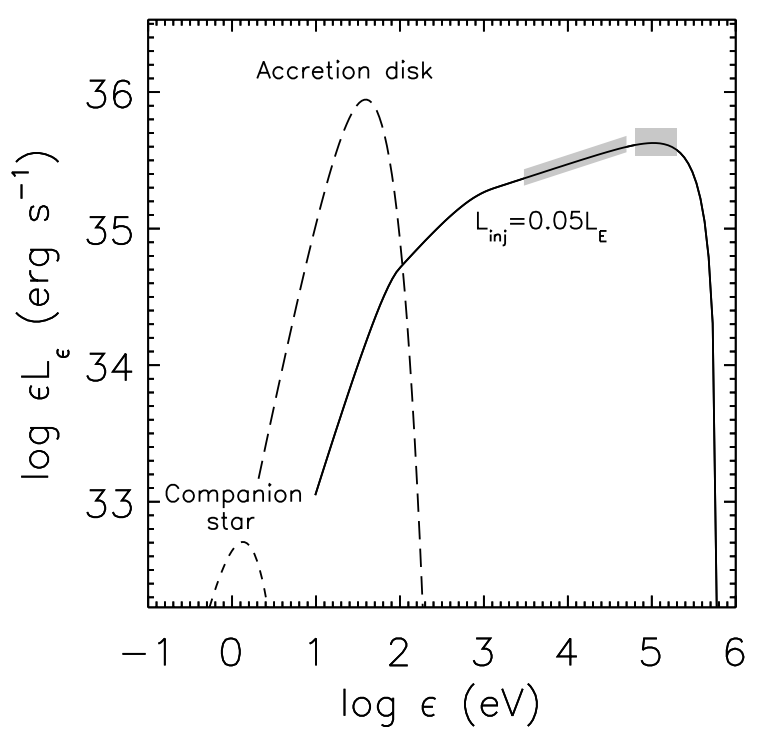

Fig. 1. A system similar to XTE J1118+480. The short and long dashed lines represent black bodies with characteristics similar to the companion star and accretion disk respectively. The solid line is the accretion disk seed photon ECS emission for $L_{\mathrm{inj}}=0.05 L_{\mathrm{E}}$. The shaded area corresponds to observations (McClintock et al. 2001b) taken in the hard state.

$p=2 \alpha=1.6$, and $\gamma_{2} \approx\left(\epsilon_{\text {peak }} / \epsilon_{0}\right)^{1 / 2} \approx 65$. We assume $\mathcal{D} \approx 1$, because the flow velocity is unknown. The X-ray power spectrum of XTE J1118+480 cuts off at frequencies $f \gtrsim 100 \mathrm{~Hz}$ (Revnivtsev et al. 2000). This constrains $R$ to be $R \gtrsim c / f \approx 10^{8} \mathrm{~cm}$, and we set $R=10^{8} \mathrm{~cm}, d=R$.

Based on the extremely weak reflection features of the source, we model the X-ray SED as jet EC emission. This requires a jet power $L_{\text {inj }}=0.05 L_{\mathrm{E}}$, a value higher by a factor of $\sim 10$ from the lower limit $L_{\mathrm{inj}}$. The solid line in Fig. 1 shows the model SED and the shaded area corresponds to observations (McClintock et al. 2001b). The cooling time $t_{\mathrm{c}}$ and length $l_{\mathrm{c}}$ of the X-ray emitting electrons are $t_{\mathrm{c}} \sim 10 \mathrm{~ms}, l_{\mathrm{c}} \sim 10^{8} \mathrm{~cm}$. Note the cooling spectral break at $\epsilon_{\mathrm{b}} \approx 1 \mathrm{keV}$, in agreement with a similar observed break (McClintock et al. 2001b).

\subsection{A microquasar with a high mass companion}

The prototype of this class is Cygnus X-1. This microquasar has an O9.7 Iab supergiant companion, luminosity $L_{\mathrm{s}} \approx 9.6 \times 10^{38} \mathrm{erg} \mathrm{s}^{-1}$ (Herrero et al. 1995). The separation between the $\mathrm{O}$ star and the massive black hole $\left(\mathrm{M} \approx 10 M_{\odot}\right)$ is $R_{\mathrm{s}} \approx 2.8 \times 10^{12} \mathrm{~cm}$. Cygnus $\mathrm{X}-1$ in its hard state displays a soft X-ray excess, which is attributed to a truncated accretion disk. Assuming a distance of $2 \mathrm{kpc}$ implies $L_{\mathrm{d}} \approx 7.3 \times 10^{36} \mathrm{erg} \mathrm{s}^{-1}$ peaking at $\sim 130 \mathrm{eV}$ (Bałucińska-Church et al. 1995). The SED peaks at $\sim 100 \mathrm{keV}$ and has a luminosity $L_{\text {peak }} \approx 1.5 \times$ $10^{37} \mathrm{erg} \mathrm{s}^{-1}$ (McConnell et al. 2000; Di Salvo et al. 2001); the spectral index $\alpha \approx 0.8$ corresponds to $p=1.6$.

The power spectrum of Cygnus X-1 peaks at $f \approx$ $0.1 \mathrm{~Hz}$ (Sunyaev \& Revnivtsev 2000). For modelling purposes we adopt a source size $R=c / f \approx 10^{11} \mathrm{~cm}$. We also 


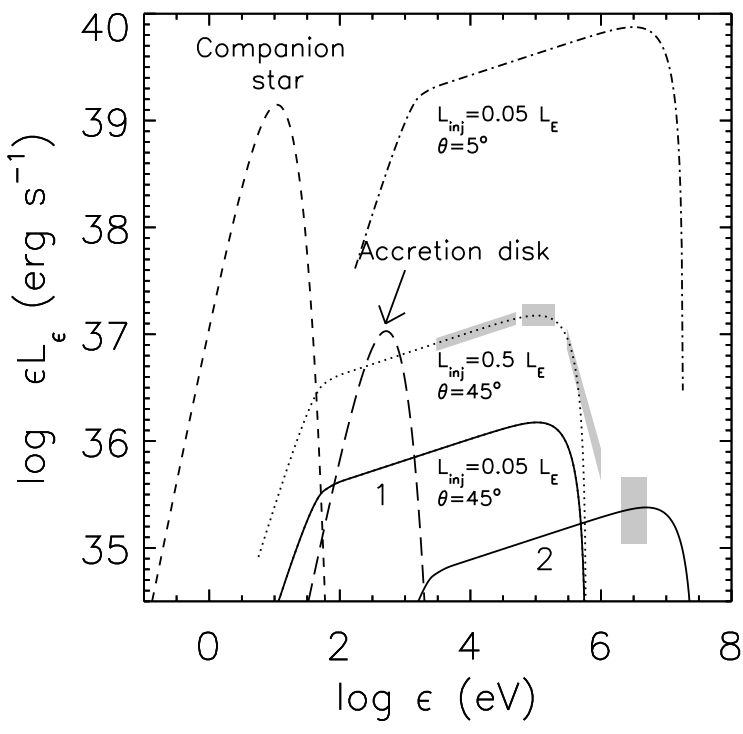

Fig. 2. A system similar to Cygnus X-1. The short and long dashed lines represent black bodies with characteristics similar to the companion star and accretion disk respectively. The ECS emission due to the star (1) and accretion disk (2) seed photons is plotted for an observing angle $\theta=45^{\circ}$ and $L_{\text {inj }}=0.05 L_{\mathrm{E}}$ (solid lines). The ECS contribution due to the companion star is also plotted for $\theta=45^{\circ}$ and $L_{\mathrm{inj}}=0.5 L_{\mathrm{E}}$ (dotted line), and for $\theta=5^{\circ}$ and $L_{\text {inj }}=0.05 L_{\mathrm{E}}$ (dot-dashed line). The Lorentz factor of the flow is $\Gamma=3$. The shaded area corresponds to observations (McConnell et al. 2000; Di Salvo et al. 2001).

set the distance $d_{\mathrm{a}}$ from the base of the jet to the accretion disk $d_{\mathrm{a}}=R$. The distance of the jet base from the star is therefore approximately equal to the orbital separation, $d=R_{\mathrm{s}}$. Under these conditions the companion star photon field dominates over the accretion disk by a factor of $\sim 10$. The Lorentz factor of the bulk motion is set to $\Gamma=3$ and the viewing angle to $\theta=45^{\circ}$, in agreement with observations (Stirling et al. 2001). This corresponds to $\mathcal{D}=1$. The maximum electron energy that corresponds to $\epsilon_{0}$ is then $\gamma_{2} \approx\left(\epsilon_{\text {peak }} / \epsilon_{0}\right)^{1 / 2}=165$.

In Fig. 2 we plot the model SED (solid lines) for $L_{\text {inj }}=0.05 L_{\mathrm{E}}$, a value which is higher by a factor of $\sim 10$ than the lower limit inferred by Fender (2001a). As can be seen, the model flux is $\sim 10$ times lower than the X-ray observed flux (McConnell et al. 2000; Di Salvo et al. 2001). Attributing the jet luminosity solely to ECS jet emission requires a high value of $L_{\mathrm{inj}}=0.5 L_{\mathrm{E}}$. However, the strong reflection features in the hard state spectrum of Cygnus X1 strongly suggest that ECS does not dominate the X-ray flux and that the actual jet power is $L_{\mathrm{inj}}<0.5 L_{\mathrm{E}}$. For the plausible value $L_{\text {inj }}=0.05 L_{\mathrm{E}}$ used here, the $\sim$ few $\mathrm{MeV}$ emission of Cygnus X-1 is explained as ECS of accretion disk photons. Note that for a Cygnus X-1 - like system pointing toward us, the condition that the jet does not contribute significantly to the observed X-ray flux corresponds to $L_{\text {inj }} \ll 10^{-5} L_{\mathrm{E}}$, a value much lower than the inferred minimum.

\subsection{Ultraluminous $X$-ray sources}

Variable and, therefore, compact X-ray sources of luminosity up to $\sim 10^{41} \mathrm{erg} \mathrm{s}^{-1}$ have been observed in nearby galaxies (Makishima et al. 2000; Fabbiano et al. 2001). These sources are displaced from the galactic centre and, if they radiate isotropically, indicate a black hole mass $M \geq 50-100 M_{\odot}$. However, it is difficult to understand how such systems could be formed in the required number, which has led King et al. (2001) to the conclusion that the emission may, in fact, be beamed, a view supported by a recent X-ray population synthesis study (Koerding et al. 2002).

Beaming immediately suggests a connection with the model presented above of nonthermal ECS from a relativistic jet in a microquasar. The peak luminosity of ECS appears amplified due to Doppler boosting by a factor of $\mathcal{D}^{5}$ for a continuous jet. Even using the mildly relativistic jet parameters appropriate for Cygnus $\mathrm{X}-1 \quad(\Gamma=3$, $\mathcal{D}_{\text {max }} \approx 5$.8) leads to a substantial maximum amplification factor $\approx 7 \times 10^{3}$. Such a source could reach an apparent luminosity $\sim 2 \times 10^{40} \mathrm{erg} \mathrm{s}^{-1}$. To illustrate this point, we plot in Fig. 2 the model SED for an observer at an angle $\theta=5^{\circ}$ to a jet with $L_{\mathrm{inj}}=0.05 L_{\mathrm{E}}$.

ULXs are seen in both hard and soft states and exhibit transitions from a soft to a hard state and vice-versa (Kubota et al. 2001; La Parola et al. 2001). If ULXs are indeed microquasars similar to Cygnus X-1, both their soft and hard states cannot be due to thermal and/or coronal emission, since these mechanisms, even at Eddington efficiency, cannot produce more than $\sim 10^{39} \mathrm{erg} \mathrm{s}^{-1}$ for a $\sim 10 M_{\odot}$ black hole. This is in agreement with our picture for the beamed hard state, where, in a manner similar to that of blazars, the thermal and/or coronal X-ray flux is swamped by the jet emission and no reflection features should be observed in their $\mathrm{X}$ ray spectra. The lack of observed reflection features in ULXs is confirmed not only for the hard, but also for the soft state (Makishima et al. 2000; Kubota et al. 2001; La Parola et al. 2001). The luminosity and lack of reflection features in the soft state of ULXs suggests by implication that their soft X-ray flux must also be beamed, so that a relativistic jet must still exists in the soft state. This soft-state jet, in analogy to the hard-state jet, would not make a significant contribution to the soft state $\mathrm{X}$ ray spectrum of an unfavourably oriented galactic microquasar similar to Cygnus X-1, where the soft state X-ray emission is dominated by thermal and coronal emission.

The idea that the ULXs are related to high companion mass microquasars is in agreement with optical observations suggesting that the ULX NGC 5204 X-1 has an O star companion (Roberts et al. 2001). Additionally, a possible X-ray periodicity has been observed in an ULX in the spiral galaxy IC 342 (Sugiho et al. 2001), which, if interpreted as orbital modulation, is compatible with emission from a microquasar with a high mass companion star. 


\section{Discussion and conclusions}

Energetic electrons in the relativistic radio jets of microquasars in the hard state up-scatter companion star and/or accretion disk photons, producing beamed X-rays. The level of this ECS emission depends on the power $L_{\text {inj }}$ of the jet. Observationally derived lower limits are $\sim 10^{-2}-10^{-3} L_{\mathrm{E}}$. However, this is an underestimate, because it does not account for adiabatic and Compton losses. XTE J1118+480 is a system with a low mass companion that shows no reflection features in its hard state X-ray spectrum. We, therefore, reproduce its X-ray flux as ECS of accretion disk photons from a jet with $L_{\mathrm{inj}}=0.05 L_{\mathrm{E}}$. Cygnus X-1, is a system with an $\mathrm{O}$ star companion. Its reflection features disfavour ECS models for the hard state X-ray spectrum. However, a jet power $L_{\text {inj }}=0.05 L_{\mathrm{E}}$ reproduces the MeV flux as ECS of accretion disk photons. It is this $\mathrm{MeV}$ excess that prompted the introduction of a non-thermal electron population in coronal models (McConnell et al. 2000). In our case, because the X-ray and $\mathrm{MeV}$ flux in the hard state are produced by different mechanisms, we expect that they will exhibit different variability characteristics.

A microquasar in the hard state can appear as an ULX in a nearby galaxy, if the jet is pointing toward us. The thermal/coronal emission is then swamped by the beamed ECS emission, resulting in the absence of reflection features, in agreement with observations. The radio flux from a system similar to Cygnus X-1, located at a distance of $\sim 2 \mathrm{Mpc}$, and pointing toward us, would be rather weak $(\sim 10 \mu \mathrm{Jy})$. The analogue of the galactic soft state depends on the existence of a jet in this state. If there is no jet, the system would be practically in an off state with a luminosity similar to that of the soft state of Cygnus X-1. However, ULXs are routinely seen in a luminous $\left(\sim 10^{40} \mathrm{erg} \mathrm{s}^{-1}\right)$ soft state, exhibiting transitions from one state to the other. Moreover, no reflection features are detected in the soft state. Therefore, if ULXs are microquasars, then a jet must exist in the soft state. The jet parameters should be different, reflecting the different state of the central engine producing the jet. The very weak soft state radio emission may be related to the higher luminosity of the accretion disk. It is possible that due to this increased luminosity, the maximum energy that electrons can be accelerated to in the soft state jet is smaller than that in the hard state jet. Most the jet power is then converted to X-ray emission through increased ECS losses, resulting in a smaller fraction of the jet power being available for radio emission.

To summarise, we demonstrate that ECS of the companion star and/or the accretion disk photons by energetic electrons in a relativistic jet contributes significantly or even dominates the X-ray flux of microquasars in the hard state. It also provides an explanation of the $\mathrm{MeV}$ emission as ECS from accretion disk seed photons. Finally, we argue that the model explains ULX in nearby galaxies as beamed microquasars similar to Cygnus X-1.
Acknowledgements. Part of this work was supported by the EU TMR programme under contract FMRX-CT98-0168.

\section{References}

Atoyan, A. M., \& Aharonian, F. A. 1999, MNRAS, 302, 253

Bałucińska-Church, M., Belloni, T., Church, M. J., \& Hasinger, G. 1995, A\&A, 302, L5

Band, D. L., \& Grindlay, J. E. 1986, ApJ, 311, 595

Corbel, S., Fender, R. P., Tzioumis, A. K., et al. 2000, A\&A, 359,251

Dermer, C. D. 1995, ApJ, 446, L63

Di Salvo, T., Done, C., Życki, P. T., Burdere, L., \& Robba, N. R. 2001, ApJ, 547, 1024

Fabbiano, G., Zezas, A., \& Murray, S. S. 2001, ApJ, 554, 1035

Fender, R. P., \& Pooley, G. G. 2000, MNRAS, 318, L1

Fender, R. P. 2001, MNRAS, 322, 31

Georganopoulos, M., Kirk, J. G., \& Mastichiadis, A. 2001, ApJ, 561,111

Herrero A., Kudritzki, R. P., Gabler, R., Vilchez, J. M., \& Gabler, A. 1995, A\&A, 297, 556

Hjellming, R. M., \& Johnston, K. J. 1988, ApJ, 328, 600

King, A. R., Davies, M. B., Ward, M. J., Fabbiano, G., \& Elvis, M. 2001, ApJ, 552, L109

Koerding, E., Falcke, H., \& Markoff, S. 2002, A\&A, 382, L13

Kubota, A., Mizuno, T., Makishima, K., et al. 2001, ApJ, 547, L119

La Parola, V., Peres, G., Fabbiano, G., Kim, D. W., \& Bocchino, F. 2001, ApJ, 556, 47

Levinson, A., \& Blandford, R. 1996, ApJ, 456, L29

Makishima, K., Kubota, A., Mizuno, T., et al. 2000, ApJ, 535, 632

Markoff, S., Falcke, H., \& Fender, R. P. 2001, A\&A, 372, L25

Matsumoto, H., Tsuru, T. G., Koyama, K., et al. 2001, ApJ, 547, L25

McConnell, M. L., Ryan, J. M., Collmar, W., et al. 2000, ApJ, 543,928

McClintock, J. E., Garcia, M. R., Caldwell, N., et al. 2001a, ApJ, 551, L147

McClintock, J. E., Haswell, C. A., Garcia, M. R., et al. 2001b, ApJ, 555, 477

Miller, J. M., Ballantyne, D. R., Fabian, A. C., \& Lewin, W. H. G. 2002, MNRAS, submitted [astro-ph/0111027]

Mirabel I. F., \& Rodríguez L. F. 1999, ARA\&A, 37, 400

Paredes, J. M., Martí, J., Ribó, M., \& Massi, M. 2000, Science, 288,2340

Revnivtsev, M., Sunyaev, R., \& Borozdin, K. 2000, A\&A, L37

Roberts, T. P., Goad, M. R., Ward, M. J., et al. 2001, MNRAS, 325, L7

Sams, B. J., Eckart, A., \& Sunyaev, R. 1996, Nature, 382, 47

Stirling A. M., Spencer, R. E., de la Force, C. J., et al. 2001, MNRAS, 327, 1273

Sugiho, M., et al. 2001, ApJ, 561, L73

Sunyaev, R., \& Revnivtsev, M. 2000, A\&A, 358, 617

Zdziarski, A. 2000, IAU Symp., 195, ed. P. C. H. Martens et al. (San Francisco: ASP), 153 